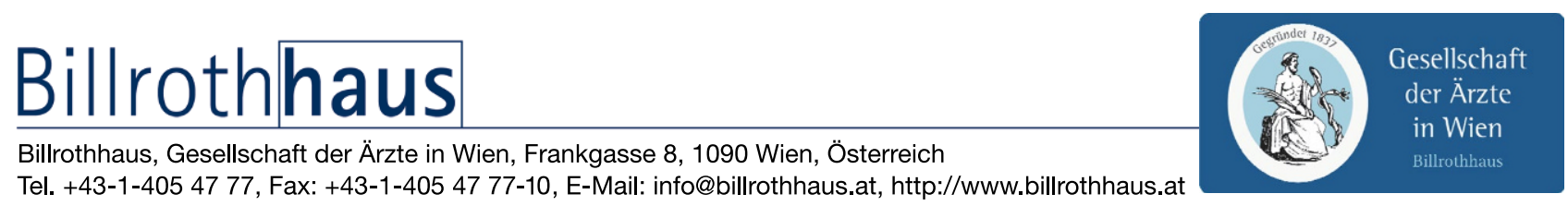

\section{Veranstaltungs- tipps:}

\author{
Medizin \& Geschichte: Medical \\ History Tour \\ Termine: 27.09., 21.10., 18.11., \\ 16.12.2021, 15.00 Uhr
}

Medizin aktuell: DSGVO für Ärztlnnen \& Co: Leitlinien zur DatenschutzCompliance im Gesundheitswesen Termin: 06.10.2021, 19.00 Uhr

Medizin \& Geschichte: 200 Jahre Rudolf Virchow. Errungenschaften, Kontroversen und Irrtümer Termin: 13.10.2021, $18.00 \mathrm{Uhr}$

Medizin aktuell: COVID-19 Update im Billrothhaus - Meet the Experts Termin: 12.10.2021, 19.00 Uhr

Medizin aktuell: Zum 60. Jahrestag der modernen Parkinsontherapie Termin: 10.11.2021, 19.00 Uhr

Medizin \& Geschichte: Virtuelles Kamingespräch „Facts and Fakes“ (Vorprogramm zum Symposium "Lust und Seuche" am 12.11.) Termin: 11.11.2021, 19.00 Uhr

Medizin \& Geschichte: Lust und Seuche - von Paracelsus bis Anthony Fauci (mit anschließender Ausstellungseröffnung) Termin: $12.11 .2021,9.00 \mathrm{Uhr}$
Alle Detailprogramme finden Sie auf www.billrothhaus.at oder via QR-Code:

\section{Medizinische Universität Wien und Gesellschaft der Ärzte in Wien rufen Dora Brücke-Teleky Award ins Leben}

In einer gemeinsamen Initiative zur Förderung und Würdigung junger Wissenschaftlerlnnen und ihrer Leistungen rufen die Medizinische Universität Wien und die Gesellschaft der Ärzte in Wien einen Preis für herausragende Postdoc Papers ins Leben: Der Dora BrückeTeleky Award wird erstmals im Herbst 2021 verliehen.

Dora Brücke-Teleky war eine der ersten Frauen, die in Wien Medizin studierten. Sie promovierte 1904 an der Medizinischen Fakultät der Universität Wien und spezialisierte sich in der Folge auf Gynäkologie und Urologie. Im
Jahr 1911 wurde sie als erste Frau in die Gesellschaft der Ärzte in Wien aufgenommen. Aufgrund des „Anschlusses" emigrierte sie 1938 in die USA und praktizierte dort bis in die 1950er Jahre.

Der Dora Brücke-Teleky Award wird künftig zweimal jährlich von einem Komitee, bestehend aus Angehörigen der MedUni Wien, an herausragende, jeweils im Vorjahr erschienene Papers von Postdocs der MedUni Wien verliehen. Teilnahmeberechtigt sind Postdoc Wissenschaftlerlnnen aus allen Disziplinen, die an der MedUni Wien angestellt sind.

Die ausgewählten Kandidatlnnen werden ihre Arbeit im Rahmen einer Veranstaltung abwechselnd im Van Swieten Saal der MedUni Wien und im Billrothhaus der Gesellschaft der Ärzte in Wien präsentieren. Der Award selbst besteht aus einem Zertifikat und einem Gutschein für eine kulturelle Veranstaltung in Wien.

Als Kick-off-Event für den Award laden die beiden Organisationen am Mittwoch, den 24. November 2021, zu einer Vorlesung ein, die auch den Auftakt zu einer gemeinsamen Veranstaltungsreihe bilden wird. Nähere Informationen folgen in Kürze.

Publisher's Note Springer Nature remains neutral with regard to jurisdictional claims in published maps and institutional affiliations. 